\title{
THE ROLE OF SPACE CHARGE IN THE PERFORMANCE OF THE BUNCHING SYSTEM FOR THE ATLAS POSITIVE ION INJECTOR*
}

\author{
R. C. Pardo, Argonne National Laboratory, Argonne, IL 60439 USA \\ and R. Smith, Kalamazoo College, Kalamazoo, MI 49006 USA
}

The bunching system of the ATLAS Positive Ion Injector consists of a four-frequency harmonic buncher, a beam-tail removing chopper, and a $24.25 \mathrm{MHz}$ spiral resonator sine-wave rebuncher. The system is designed to efficiently create beam pulses of approximately $0.25 \mathrm{nsec}$ FWHM for injection into and acceleration by the ATLAS superconducting linac. Studies of the effect of space charge on the performance of the system have been undertaken and compared to simulations as part of the design process for a new bunching system to be developed for a second ion source. Results of measurements and modeling studies indicate that the present system suffers significant bunching performance deterioration at beam currents as low as 5 e $4 \mathrm{~A}$ for ${ }^{238} U^{26+}$ at a velocity of $\beta=0.0085$. The low beam current tolerance of the present system is in reasonable agreement with computer simula-tions. Studies of two alternatives to the present bunching system are discussed and their limitations are explored.

\section{INTRODUCTION}

The ATLAS Superconducting Heavy-Ion Linear Accelerator[1,2] was designed with the goal of providing high quality beams of any heavy-ion species for fundamental research in nuclear and atomic physics. Beam currents were expected to be large by historical standards; as high as ten to a hundred particle nanoamps for most beams. The effects of

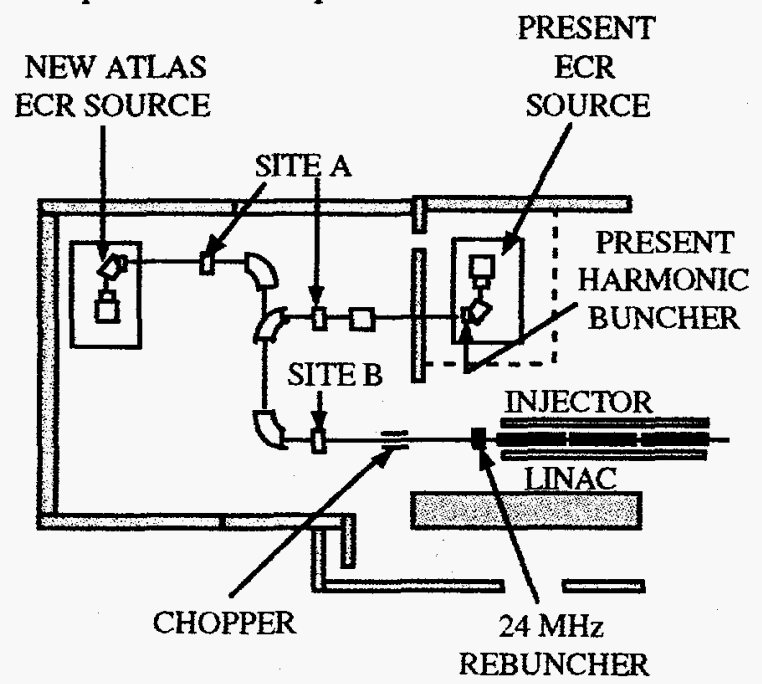

Figure 1: Floor plan of Low-Energy Beam Transport system for PII-ATLAS showing the relevant bunching system components. space charge on beam optics was considered and found not to be significant for the transverse dimensions. The effect of space charge on longitudinal bunching is more serious and observable defocusing effects were expected for beam currents of a few electrical microamps. Figure 1 shows the floor plan of the low-energy beam transport (LEBT) for the ATLAS Positive Ion Injector(PII) and indicates the location of the present bunching components.

The first stage of bunching for the PII is a four-harmonic gridded-gap buncher[3] located on a $300 \mathrm{kV}$ platform with the ECR ion source. The fundamental frequency of this buncher is $12.125 \mathrm{MHz}$ (period of $82.4 \mathrm{~ns}$ ) and a sawtoothlike wave is formed using three additional harmonics. Typical maximum bunching voltage requirements are approximately 700 volts. This bunching system is operated in such a way as to form a waist at the chopper plates to minimize the longitudinal emittance growth caused by the chopper. The beam is then further compressed for injection into the linac by a second, sine-wave buncher operating at $24.25 \mathrm{MHz}$. For beam currents of 1 e $\mathrm{AA}$ or less, bunch widths significantly less than 1 ns FWHM have been possible for a variety of beams using only the harmonic buncher. As the beam current is increased to a few microamps the bunch width at the time focus worsens as shown in Figure 2 for a beam of ${ }^{16} \mathrm{O}^{3+}$. For these studies, ${ }^{16} \mathrm{O}^{3+}$ was chosen because it is a beam with a charge-to-mass ratio (Q/A) typical of many of the heavy-ion beams accelerated at ATLAS.

The worsening of the best possible time width as beam current increases is from the repulsive space-charge force on the compressing beam. In this simple situation, the spacecharge force acts simply as a continuous defocusing lens slowing the compression process. In the beam center-ofmass system, the maximum velocity which must be imparted to an ion so as to achieve a time focus at the chopper plates is $4073 \mathrm{~m} / \mathrm{s}$. This corresponds to an ion energy in the centerof-mass of $1.37 \mathrm{eV}(0.46 \mathrm{~V})$ for ${ }^{16} \mathrm{O}^{3+}$. The repulsive spacecharge potential for a beam bunch of these particles corresponding to an average beam current of 1 e $\mu \mathrm{A}$ is $0.47 \mathrm{~V}$ for a spherical geometry. One sees immediately that the beam will become essentially a 'parallel' beam under these conditions. For higher beam currents, the waist condition will be destroyed and cannot be achieved under any condition with a fixed geometry. In the laboratory frame, these small voltages translate into a buncher voltage of 675 volts indicating that space charge forces are significant at currents as low as 1 euA. These estimates are in good agreement with the observed typical 700 volts focusing force mentioned previously.
The submitted manuscript has been authored by a contractor of the U.S. Government under contract No. W-37-109ENG-38. Accordingly, the U.S. Government retains a nonexclusive, royalty-free license to publish or reproduce the published form of this contribution, or allow others to do so, for U.S. Government purposes. 


\section{DISCLAIMER}

This report was prepared as an account of work sponsored by an agency of the United States Government. Neither the United States Government nor any agency thereof, nor any of their employees, makes any warranty, express or implied, of assumes any legal liability or responsibility for the accuracy, completeness, or usefulness of any information, apparatus, product, or process disclosed, or represents that its use would not infringe privately owned rights. Reference herein to any specific commercial product, process, or service by trade name, trademark, manufacturer, or otherwise does not necessarily constitute or imply its endorsement, recommendation, or favoring by the United States Government or any agency thereof. The views and opinions of authors expressed herein do not necessarily state or reflect those of the United States Government or any agency thereof. 


\section{DISCLAIMER}

Portions of this document may be illegible in electronic image products. Images are produced from the best available original document. 


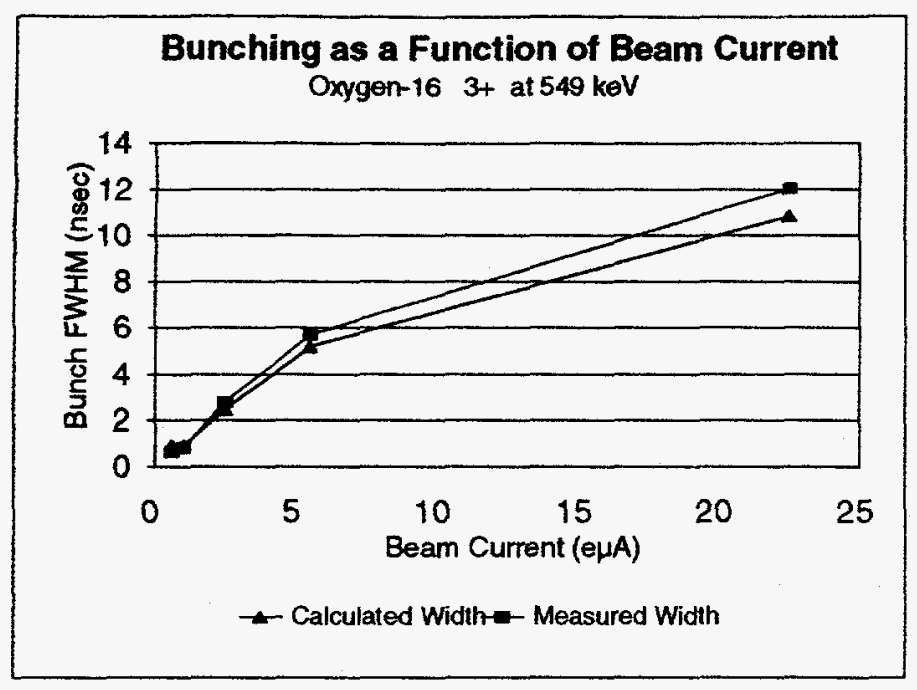

Figure 2: Observed beam bunch width at PII entrance waist location compared to performance predicted by TRACE3D.

A more quantitative comparison to observation has been made using the first-order matrix program TRACE3D[4] which can include space charge effects. The comparison to the experimental data is shown in Figure 2. These calculations confirmed the effects discussed above and showed that as the beam current increases above 1-2 enA significant and unrecoverable changes occur to the beam phase ellipse making the match into the linac much poorer. At currents much above 20 e $\mu \mathrm{A}$ the beam mismatch to the linac causes significant deterioration in the efficiency of acceleration and beam loss. The effective longitudinal emittance is also significantly worsened by the bunching system, mostly due to the nonlinear bunching of the second sine-wave buncher when the beam is spread over a phase width of approximately 180 degrees.

\section{DESIGN STUDY}

A second high charge-state ECR ion source is under construction for ATLAS and in conjunction with that project a study of the injection bunching system has been undertaken with the goal of improving the bunching performance for the high current and high charge-state beams anticipated from the new ECR ion source. TRACE3D was used to model the performance of the systems studied with regard to space charge limitations. A proprietary ray-tracing program was used to study the models with regard to resonator field profiles. The ray-tracing program did not include space charge effects, but did include the exact waveforms of the harmonic and sine-wave bunchers.

The location of the new source, injection geometry and relationship to the existing source and LEBT are indicated in Figure 1. The obvious goal is to create a bunching system which requires a much shorter focal length buncher, necessitating higher bunching voltages (shorter longitudinal focal lengths). Such a requirement must be achieved while providing good ion species resolving power so as to deliver pure beams to the linac while maintaining high beam quality, especially low longitudinal emittance.

Two locations were studied as possible sites for the harmonic buncher in the system. These are indicated in Figure 1 as 'Site $A$ ' and 'Site B'. 'Site $A$ ' is off the high voltage platform, and provides improved bunching characteristics for beams of moderate intensity. For example with a beam of $\mathrm{Q} / \mathrm{A}=0.1875$, bunching is adequate up to average currents of $40 \mathrm{e} \mu \mathrm{A}$. Average beam currents as high as 300 to 500 e $\mu \mathrm{A}$ are possible from the new ECR ion source. This location is still inadequate for beams of such intensity. In addition, the species selectivity of the magnetic analysis system in the LEBT is significantly reduced by the energy spread created by the harmonic buncher at 'Site $A$ '. The $\mathrm{Q} / \mathrm{A}$ selectivity of the transport system without the buncher operating in the present configuration is approximately 1 in 2000 . Including the energy spread from the harmonic buncher, in the present location, the selectivity in $\mathrm{Q} / \mathrm{A}$ is reduced to 1 in 400 . By moving the harmonic buncher to Site $A$ that resolution is further reduced to 1 in 200. Additionally, the need to tune the 180 degree achromat for good optical conditions adds some complexity to the everyday operation of the LEBT.

Therefore the performance of the buncher system with the harmonic buncher located at 'site B' was studied. This site might seem the most obvious choice, since the buncher focal length is the shortest and therefore the bunching electric field is the highest. But the high bunching electric fields required for this location and necessary to reduce the effects of space charge on the longitudinal optics are more difficult to obtain. A high premium on the quality of the bunching waveform is also required for these high bunching fields. In order to obtain good bunch widths for injection into the linac and to minimize the emittance growth due to the buncher

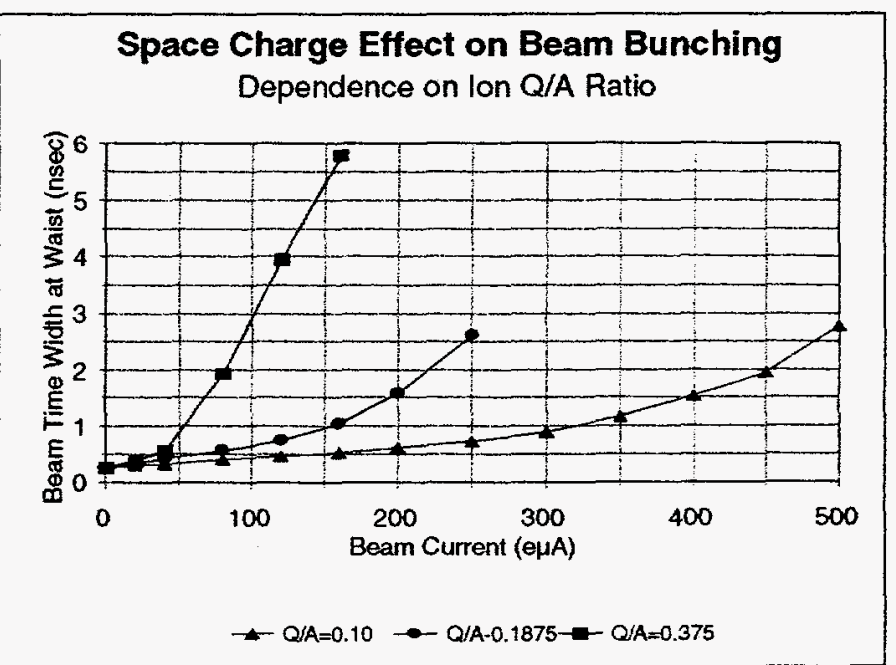

Figure 3: 'Site $B$ ' configuration. Beam bunch width versus beam current for beams with differing $Q / A$ for the harmonic buncher only. Compare the present system performance in Figure 1 to the $\mathrm{Q} / \mathrm{A}=0.1875$ curve. 
waveform, a very careful optimization of the four harmonics forming the sawtooth-like harmonic buncher waveform was found to be necessary.

The calculated results for the 'Site B' harmonic buncher location are shown in figure 3, 4, and 5. Careful optimization of the buncher waveform resulted in an empirical harmonic mix of :

$$
\begin{aligned}
& E_{b}=A \cdot(\sin (\omega t)-0.3814 \cdot \sin (2 \omega t)+ \\
& 0.1506 \cdot \sin (3 \omega t)-0.0405 \cdot \sin (4 \omega t))
\end{aligned}
$$

The barmonics found to provide optimum bunching at this location are not universal and in fact make some use of the $24.25 \mathrm{MHz}$ sine-wave second buncher waveform to provide the best total effective bunching voltage.

The reduced sensitivity of buncher performance on beam current can most immediately be seen by comparing the calculated time width versus beam current for $Q / A=0.1875$ $\left({ }^{16} \mathrm{O}^{3+}\right)$ to the present buncher performance shown in Figure 2. The 'site B' configuration makes it possible to bunch 200 e $\mu \mathrm{A}$ beams better than 3 e $\mathrm{A}$ beams with our present configuration. For low $\mathrm{Q} / \mathrm{A}$ beams such as uranium, adequate bunching at currents from 300 to $450 \mathrm{e} \mu \mathrm{A}$ is possible. For lighter mass, high charge state ions, the beam current limit is approximately $100 \mathrm{e} \mu \mathrm{A}$. The strong $\mathrm{Q} / \mathrm{A}$ dependence of bunching performance is apparent in Figures 3 and 4. In general one can characterize 'Site B' as able to handle beam currents which are a factor of 100 greater than the present system can accept.

The beam ellipse is transformed by the defocusing effect of space charge, turning a waist condition into a "parallel beam' condition. This is shown indirectly by noting the decrease in energy spread of the beam ellipse as the current increases in Figure 4, which corresponds to the increasing time width portrayed in Figure 3 . Thus the original matching condition into the linac is modified drastically and eventually the beam ellipse falls out of the acceptance ellipse of the linac. With the site $B$ configuration this does not occur until beam currents well in excess of $100 \mathrm{e} \mu \mathrm{A}$ are reached for any ion species.

The efficiency of converting the DC beam from the source into beam bunches matched into the linac acceptance is approximately $60 \%$ for our present configuration. The model calculations predict that the 'Site B' configuration of the bunching system will achieve a bunching efficiency of $65-70 \%$ acceptance. It is possible to operate the harmonic buncher in a mode which creates a virtual waist for the 24.25 $\mathrm{MHz}$ buncher. This mode requires lower buncher voltages and relaxes the requirements on waveform quality, but the bunching efficiency decreases to only approximately $40 \%$. Therefore we do not anticipate using this bunching mode.

The 'Site B' configuration appears to be a good solution to bigh current injection into the ATLAS linac. This configuration will be implemented as part of the second ECR construction project. Operation of this new configuration is expected in 1997.

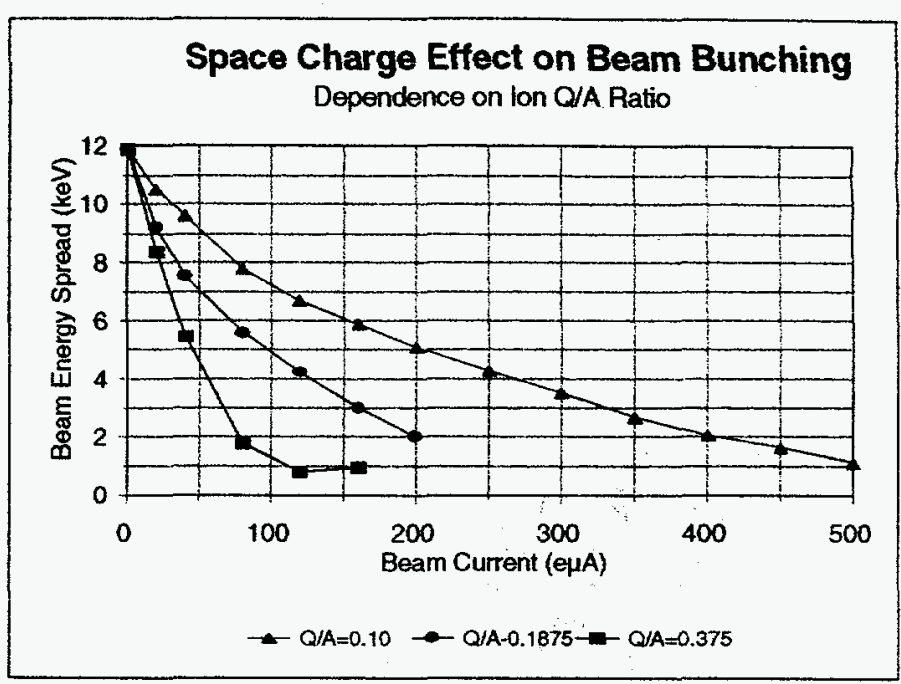

Figure 4: 'Site B' configuration. Beam energy spread for waist condition at chopper versus beam current for beams with differing $\mathrm{Q} / \mathrm{A}$.

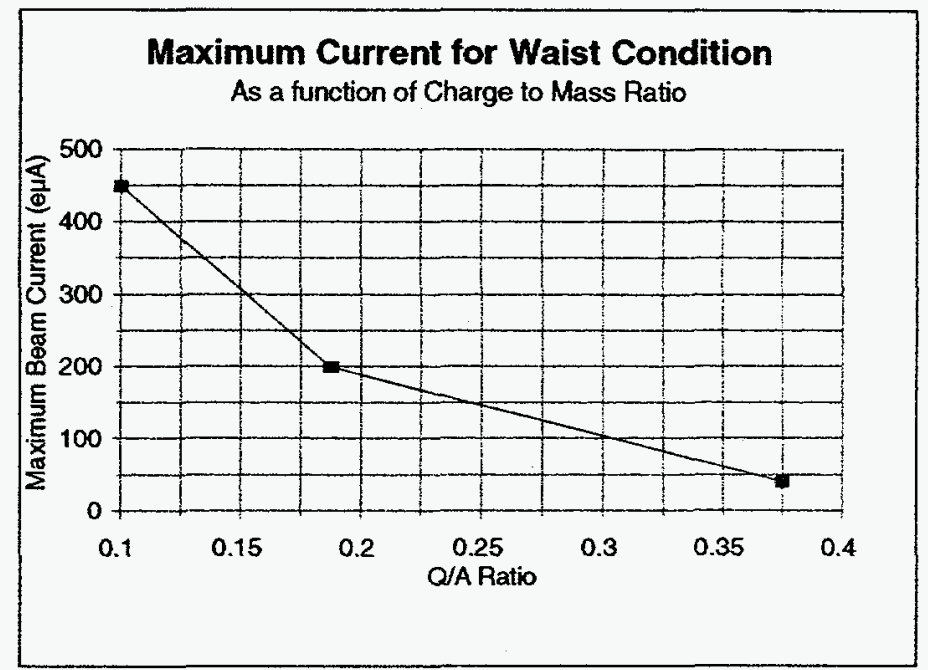

Figure 5: The maximum beam current for which a true beam waist can be achieved at the chopper as a function of $Q / A$ for the Site $B$ configuration.

*Supported by US D.O.E., Nucl. Phys. Div., contract W-31109-ENG-38.

\section{REFERENCES}

[1] R. C. Pardo, L. M. Bollinger, and K. W. Shepard, Nucl. Instrum. and Methods, B24/25, 746(1987).

[2] L. M. Bollinger, et al., Nucl. Instrum. and Methods, B79, 753(1993).

[3] F. J. Lynch, R. N. Lewis, L. M. Bollinger, and O. D. Despe, Nucl. Instrum. and Methods, 159, 245(1979).

[4] K. R. Crandall, "Trace 3-D Documentation", Los Alamos National Laboratory, LA-11054-MS, (August 1987). 